\title{
Meilensteine auf dem Weg zur heutigen Kardiologie
}

\author{
Roger Hullina, Beat Kaufmann ${ }^{b}$ Michael Kühne ${ }^{c}$, Hans Ricklid, Peter Wenawesere, Michael Zellweger \\ a Prof. Dr. med., Präsident der Arbeitsgruppe Herzinsuffizienz der SGK, CHUV Lausanne \\ b Prof. Dr. med., Präsident der Arbeitsgruppe Echokardiographie und Cardiac Imaging der SGK, Universitätsspital Basel \\ c PD Dr. med., Mitglied der Arbeitsgruppe Herzschrittmacher und Elektrophysiologie der SGK, Universitätsspital Basel \\ d Prof. Dr. med., Präsident des Steering Committee AMIS Plus, Kantonsspital St. Gallen \\ e Prof. Dr. med., Präsident der Arbeitsgruppe interventionelle Kardiologie der SGK, Klinik im Park, Zürich \\ ${ }^{\dagger}$ Prof. Dr. med., Präsident der SGK, Universitätsspital Basel
}

Herz-Kreislauf-Erkrankungen betreffen angesichts der demographischen Entwicklung zunehmend mehr Menschen und stellen in der Schweiz die häufigste Todesursache dar. Alleine die Hospitalisationen wegen Herz-Kreislauf-Krankheiten betrafen 2014114000 Personen. Von welchem Nutzenzuwachs der letzten Jahrzehnte in der Kardiologie diese und andere Patienten profitieren können, wird anhand verschiedener Beispiele dargestellt.

\section{Schonendere, schnellere und exaktere Methoden ermöglichen genauere Diagnosen und effektivere Therapien}

Herzultraschalluntersuchungen haben die moderne kardiovaskuläre Medizin massgeblich mitgeprägt. Was früher mit dem Stethoskop vermutet wurde, kann heute mit Ultraschall-Bildgebung detailgetreu dargestellt und quantifiziert werden - und dies ohne Strahlenbelastung: Herzklappenfehler, Einschrän- kungen der Pumpfunktion des Herzens z.B. beim Herzinfarkt, Abnützung oder Infekt der Herzklappen, Flüssigkeit im Herzbeutel, Auswirkungen von Systemerkrankungen auf das Herz. Diese verbesserte und auch in Notfallsituationen rasch verfügbare Diagnostik ist aus der modernen Kardiologie nicht mehr wegzudenken. Neue, schonende Therapieverfahren zur Behandlung von Klappenfehlern, welche einer zunehmend älteren Bevölkerung zugutekommen, könnten ohne komplexe, teilweise auch dreidimensionale

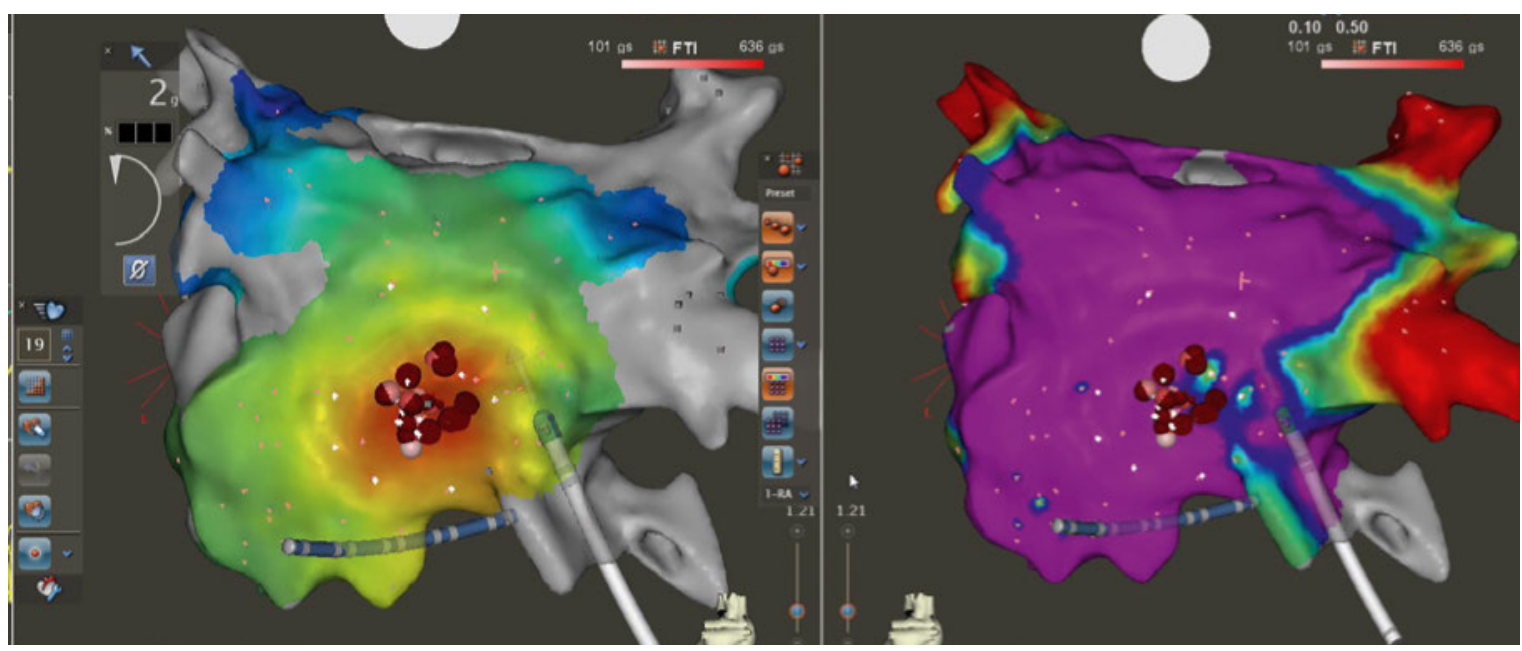

Die Kardiologie hat sich in den letzten Jahrzehnten stark entwickelt - zum Vorteil vieler Patienten. Dank Verfahren wie der Katheterablation (=Verödungsbehandlung) können heute Betroffene mit unterschiedlichsten Arten von Herzrhythmusstörungen behandelt und oft dauerhaft geheilt werden. Die Bilder zeigen eine dreidimensionale Rekonstruktion eines linken Vorhofs (von hinten) mit dem Ablationskatheter (in weiss) und einem Diagnostikkatheter (in blau). Die roten Punkte zeigen den Ort, an dem die Tachykardie mit der Ablation beendet werden konnte. Die Rekonstruktionen erlauben es, die Untersuchung praktisch ohne Röntgenstrahlung durchzuführen. 
Ultraschallbildgebung gar nicht erst durchgeführt werden.

Die Schnittbildtechniken wie Herz-MRI (Magnetresonanztomographie), Computertomographie, Szintigraphie und PET (Positronemissionstomographie) erlauben eine auf die Patienten und Fragestellung fokussierte Anwendung. Mit hoher Verlässlichkeit kann mit diesen Methoden ein Problem der Herzkranzgefässe erkannt oder ausgeschlossen werden. Mit MRI und den nuklearmedizinischen Methoden kann das Herzgewebe charakterisiert werden, so dass Entzündungen und Einlagerungen im Herzmuskel nachgewiesen werden können, wo früher eine Gewebeprobe entnommen werden musste. Diese Techniken erlauben die exakte Planung und Durchführung von Herz- und Herzklappeneingriffen, die für die Patienten weit weniger belastend sind als noch vor Jahren.

\section{Viele Herzrhythmusstörungen sind in den letzten 20 Jahren heilbar geworden}

Frau Anderegg (Name geändert) ist eine von vielen Patienten, die in den letzten Jahren in einem Schweizer Zentrum wegen ihrer Herzrhythmusstörungen behandelt wurde. Sie litt trotz medikamentöser Behandlung über Monate immer wieder unter Episoden von Herzrasen. Sie fehlte wegen ihrer Herzrhythmusstörungen häufig im Beruf als Verkäuferin und verlor ihre Anstellung. Bis Anfang der Neunziger Jahre existierten keine medizinischen Massnahmen, um der Patientin helfen zu können. Heute kann mit einer Verödungsbehandlung (Katheterablation) geholfen werden, die Rhythmusstörung der Patientin konnte geheilt werden. Die Patientin war selbst erstaunt, wie rasch sie wieder in den Alltag zurückfand. Sie ist heute wieder voll berufstätig.

Die Symptome von Herzrhythmusstörungen, die in der Schweiz tausende von Menschen betreffen, sind mannigfaltig, oft gehören Herzrasen und Unwohlsein dazu, bisweilen kommen Atemnot oder ein Bewusstseinsverlust dazu. Während früher im Wesentlichen nur die Therapie mittels Elektroschock (Elektrokonversion) und mit Medikamenten verfügbar war, welche nur die Symptome und nicht die Ursache der Krankheit behandelten und oft auch nicht zum Erfolg führten, wurden in den letzten 20 Jahren Verfahren (Katheterablation = Verödungsbehandlung) entwickelt, mit welchen heute Patienten mit unterschiedlichsten Arten von Herzrhythmusstörungen behandelt und in vielen Fällen sogar dauerhaft geheilt werden. Im Jahr 2016 wurden in der Schweiz 6431 Verödungsbehandlungen durchgeführt. Dies erfordert eine spezifische hochtechnisierte Infrastruktur im Spital. Durch den zusätzlichen Einsatz von dreidimensionalen Herzmodellen, welche mit Ultraschall oder MRI erstellt werden, sind diese Eingriffe sehr risikoarm geworden $(<1-2 \%$ ernsthafte Komplikationen).

Wenn gefährliche Herzrhythmusstörungen oder sogar ein Kammerflimmern auftreten, besteht heute die Möglichkeit, bei Patienten eine "Mini-Version" eines Defibrillators einzupflanzen. Der implantierbare Defibrillator (ICD) hat vor etwa 20 Jahren im klinischen Alltag Einzug gehalten. Heute ist die Operation durch kontinuierliche Verbesserungen zum Routineeingriff geworden und dient Patienten mit einem erhöhten Risiko für gefährliche Herzrhythmusstörungen quasi als Schutzengel, denn er kann lebensbedrohliche Herzrhythmusstörungen effektiv behandeln.

\section{Durch die eigenen Gefässe hin zum Ort des Geschehens - Risikoärmere Eingriffe eröffnen Therapieoptionen für mehr Patienten}

Dieses Jahr feiert die interventionelle Kardiologie das 40-Jahre-Jubiläum der ersten Ballondilatation durch A. Grüntzig. Diese ermöglicht, verengte oder verschlossene Herzkrankgefässe mit einem aufblasbaren Katheter wieder aufzudehnen, was vielen Patienten beim Herzinfarkt das Leben rettet oder im chronischen Stadium eine Bypass-Operation erspart. Nach dieser bahnbrechenden Erfindung und Revolution in der Behandlung der koronaren Herzkrankheit wurden weitere wichtige Erfolge gefeiert. Mittels Koronarstents konnten Gefässe dauerhaft gestützt und ihre (erneute) Verengung verhindert werden. Zudem konnte mit Hilfe lipidsenkender Medikamente vielen Herz-Kreislauf-Erkrankungen vorgebeugt werden. Diese Faktoren tragen neben vielen anderen dazu bei, dass die Lebenserwartung der Menschen in der Schweiz eine der höchsten weltweit ist. Die Lebenserwartung in der Schweiz ist in den letzten 25 Jahren bei den Männern um 6,7 und bei den Frauen um 4,1 Jahre angestiegen.

Über Jahre wurde die Bedeutung der valvulären, also der Herzklappen-Erkrankungen hinsichtlich Lebenserwartung, aber auch Lebensqualität unterschätzt. Ein Grossteil der älteren Patienten mit schweren Klappenerkrankungen wurde palliativ und nicht mittels konventioneller Herzchirurgie behandelt, weil das Risiko einer Operation am offenen Herzen zu hoch war. Die erste minimalinvasive Implantation einer Aortenklappe (TAVI) 2002 brachte den Durchbruch in der Behandlung der Herzfehler. Auch in der Schweiz konnte diese effektive Therapie schnell Fuss fassen und ist nach der Ballondilatation der zweithäufigste Eingriff in der interventionellen Kardiologie geworden (2016 
wurde 1630 Patienten so geholfen). Die Auswirkungen auf das Befinden und die Lebenserwartung der Patienten sind dabei positiv und machen den Eingriff dank seiner minimalen Invasivität äusserst attraktiv. Parallel dazu wurden in den letzten Jahren auch minimalinvasive Methoden zur Reparatur oder auch zum Ersatz der Mitralklappe entwickelt, die - beim Gesunden - einen Rückfluss des Blutes aus der linken Herzkammer in den linken Vorhof verhindert. Diese Methoden eröffnen zuvor nichtoperablen oder Hochrisikopatienten mit schwerer Mitralinsuffizienz wertvolle therapeutische Optionen (z.B. Mitraclip). Profitiert haben diese Entwicklungen von der engen Zusammenarbeit von Kardiologen und Herzchirurgen.

\section{Die Sterblichkeit bei Herzinfarkt hat stark abgenommen}

Die Diagnostik und Behandlung des Herzinfarktes hat sich in den letzten 20 Jahren extrem gewandelt. Vor 40 Jahren wurde Patienten mit Herzinfarkt eine mehrwöchige Bettruhe verordnet. Heute kann aktiv gegen die Folgen des Herzinfarktes vorgegangen werden. Das seit 1997 in Schweizer Spitälern etablierte AMIS-Plus Register mit weit über 50000 Patienten zeigt, dass insbesondere durch die Einführung der akuten Herzkatheterintervention (Akut-PCI) begleitet von verbesserten medikamentösen Massnahmen deutlich weniger Patienten im Spital an einem akuten Koronarsyndrom verstarben: bei Männern sank die Mortalität von 9,8 auf 5,5 Prozent und bei Frauen sogar von 18,3 auf 6,9 Prozent. Das Register liefert eine Fülle epidemiologischer Daten und ermöglicht eine optimale Qualitätssicherung der Spitäler insbesondere durch einen nationalen Benchmark. Die Herzinfarktmortalität in der Schweiz gehört zu den niedrigsten überhaupt dank einer sich laufend verbessernden, multidisziplinären Betreuung der betroffenen Patienten.

\section{Patienten mit ermüdetem Herzmuskel kann effektiver denn je geholfen werden}

Die Herzinsuffizienz beschreibt eine Herzschwäche mit Folgen für den gesamten Organismus. Aufgrund der reduzierten Pumpfunktion des Herzens leiden die Patienten unter Luftnot, Wasser in der Lunge, Wasseransammlungen im Körper und entwickeln mitunter weitere Organschädigungen. Die Herzinsuffizienz ist eine Volkskrankheit, die in westlichen Ländern 1,52,5\% der Bevölkerung und in der Schweiz etwa 175000 Menschen betrifft. Bei jungen Menschen ist die Erkrankung selten, jedoch sind bereits $2 \%$ der Menschen in der Altersgruppe von 55-64 Jahren, und im hohen Alter bis zu 20\% aller Menschen betroffen. Aufgrund der Überalterung der Gesellschaft dürfte sich die Anzahl der Herzinsuffizienz-Patienten in den nächsten $20 \mathrm{Jah}$ ren verdoppeln.

Intensive klinische Forschung hat in den vergangenen drei Jahrzehnten die Möglichkeiten der Herzinsuffizienz-Behandlung wesentlich verbessert. Heutzutage sind Medikamente verschiedenster Wirkklassen verfügbar, welche bei Patienten mit eingeschränkter Pumpfunktion sowohl tägliche Beschwerden und Einschränkungen als auch die Sterblichkeit vermindern. Des Weiteren ermöglichen spezifische Schrittmacher bei ausgewählten Herzinsuffizienz-Patienten die Verbesserung der Pumpfunktion. Eine Heilung ist zwar selten möglich, und das Fortschreiten der Erkrankung meist nur eine Frage der Zeit. Dennoch profitieren die betroffenen Patienten über längere Zeiträume von einer höheren Lebensqualität mit weniger Spitalaufenthalten und können ihren Alltag selbständig(er) bewältigen.

Die hier zusammengetragenen Beispiele zeigen eine "Errungenschaftsexplosion" in der Kardiologie auf, die der grossen Zahl herzkranker Menschen in der Schweiz Möglichkeiten und Perspektiven eröffnen, die vor 20 Jahren nicht denkbar waren. Kontinuierliche Forschung, Aus-, Weiter- und Fortbildung, harte Arbeit, technische Fortschritte und ein nicht nur erstklassiges, sondern auch allen zugängliches Gesundheitssystem machten diese Entwicklungen möglich.

\section{Zusammenfassung}

"Älter werden heisst auch besser werden». Dieses Zitat von Jack Nicholson charakterisiert die Entwicklungen in der Kardiologie treffend. Die Kardiologie hat sich in den letzten Jahren rasant entwickelt und Nutzen für unsere Patienten gestiftet. Mit bildgebenden Methoden können rasch exakte Diagnosen gestellt werden. Eine effiziente Beratung und Therapie unserer Patienten wird so ermöglicht. Notfallmässige Eingriffe zur Wiedereröffnung der Herzkranzgefässe bei Herzinfarkt haben die Sterblichkeit eindrücklich reduziert. Langzeitproblemen der Herzmuskelschwäche und dem Leidensdruck der Patienten kann mit neu entwickelten Medikamenten und Geräten wie Herzschrittmachern erfolgreich begegnet werden. Diese Entwicklungen fordern eine hochqualitative kontinuierliche Weiter- und Fortbildung der in der Kardiologie engagierten Ärzteschaft und den beteiligten multidisziplinären Teams.

Bildnachweis

@ Kardiologische Klinik, Universitätsspital Basel 